\title{
Shrinking Lung Syndrome: A Case Report
}

Diani Abdelwahed ${ }^{1 *}$, M Aitchtouk ${ }^{1}$, M Ouali Idrissi ${ }^{1}$, B. Boutakioute ${ }^{1}$, N Cherif Idrissi Guenouni ${ }^{1}$

${ }^{1}$ Radiology department of AR-RAZI hospital UH Mohamed VI, Cadi Ayad University Marrakech, Morocco

DOI: $10.36347 /$ sjmcr.2021.v09i02.018

| Received: 29.01.2021 | Accepted: 15.02.2021 | Published: 27.02.2021

*Corresponding author: Diani Abdelwahed

Abstract

Case Report

Shrinking lung syndrome (SLS) is a pulmonary complication of systemic lupus erythematosus (SLE) characterized by dyspnea, pleuritic chest pain, and progressive decrease in lung volumes with no evidence of pleural or interstitial disease on chest CT. We present the case of a 38-year-old female with one-year history of SLE with symptoms of shortness of breath and chest pains. A chest CT scan suggested the diagnosis of SLS which was later confirmed. Even though it's a rare condition, clinicians should have a high suspicion of SLS in patients with a long-term history of SLE and worsening dyspnea. Early treatment can be initiated to help reduce long-term morbidity and mortality and maintain the quality of life.

Keywords: Shrinking lung syndrome (SLS), systemic lupus erythematosus (SLE), A chest CT scan, quality of life. Copyright $\odot 2021$ The Author(s): This is an open-access article distributed under the terms of the Creative Commons Attribution 4.0 International License (CC BY-NC 4.0) which permits unrestricted use, distribution, and reproduction in any medium for non-commercial use provided the original author and source are credited.

\section{INTRODUCTION}

Pulmonary manifestations from systemic lupus erythematosus (SLE) affect up to $80 \%$ of patients. Shrinking Lung Syndrome (SLS) is a rare condition with an estimated prevalence of $0.5 \%$ to $1.1 \%$ in patients with SLE [1]. It is characterized by dyspnea, elevation of the diaphragmatic domes and restrictive ventilation syndrome [2, 3]. Its physiopathology remains largely unclear; however, hypotheses have been suggested, ranging from microatelectatis due to lack of surfactant and increased surface tension, diaphragm fibrosis to phrenic nerve palsy [3]. We report the case of a patient with systemic lupus whose diagnosis of SLS was made by imaging.

\section{ObServation}

A 38-year-old woman presented with chest pain and intense dyspnea. She has a history of asthma for last 20 years, and a miscarriage 8 years ago. She was diagnosed with lupus a year ago based on a bundle of clinical and immunological arguments.
The initial assessment showed no sign of infection, and the presence of a high levels of D-dimer at $1000 \mathrm{ng} / 1$.

A pulmonary embolism and also a COVID-19 pneumonia were suspected in light of the current pandemic situation so a CT angiography of the chest was requested.

The CTA showed the ascent of the left diaphragmatic dome with atelectasis of the pulmonary parenchyma nearby (Figure 1 and 2) and no sign of pulmonary embolism. Considering the clinical context and the result of the CT scan, the diagnosis of Shrinking lung syndrome was suggested.

An ultrasound exam of the diaphragm confirmed the kinetic disfunction of the left diaphragmatic dome. The patient subsequently benefited from functional respiratory tests which showed a restrictive syndrome with a functional vital capacity (FVC) at $42 \%$ of the theoretical and collapsing in decubitus $(-31 \%)$ all of which made it possible to confirm the diagnosis. The patient was then treated with corticosteroid with a dose of $1 \mathrm{mg} / \mathrm{kg}$. 


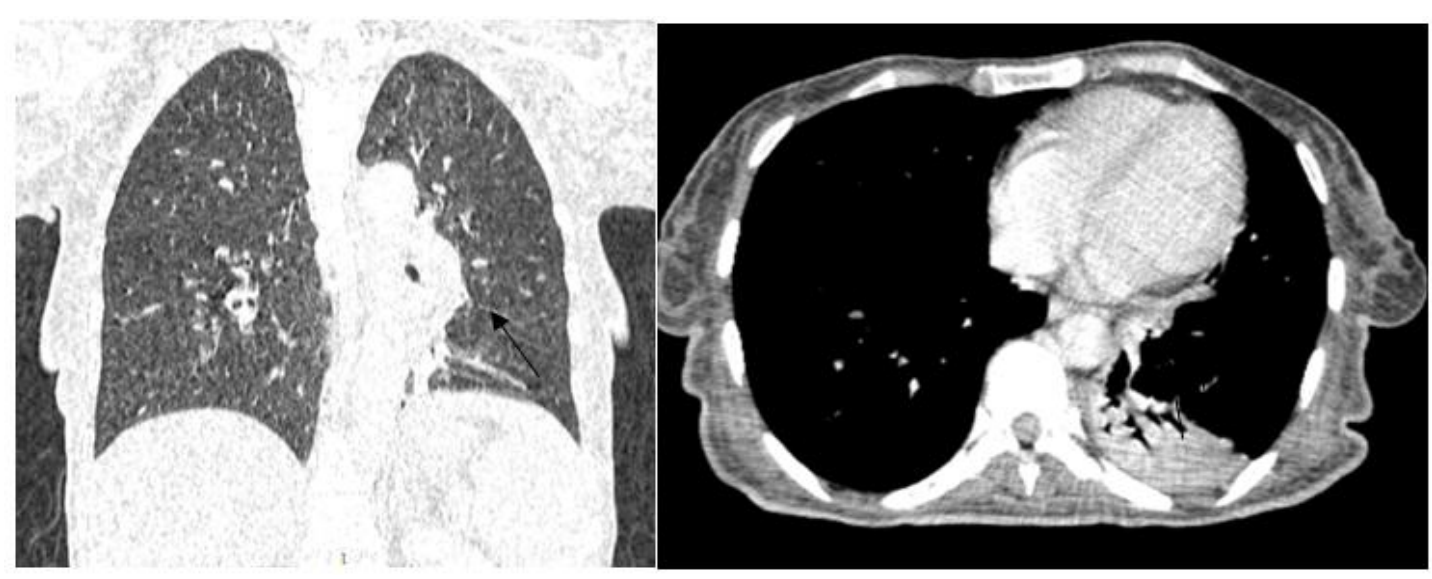

Fig-1: Thoracic CT in parenchymal window frontal reconstruction and in mediastinal window axial reconstruction showing the elevation of the left diaphragmatic dome (arrow) and atelectasis nearby (double arrow)

\section{DISCUSSION}

We report the observation of SLS, revealed by severe dyspnea and chest pain. The first description of SLS is from Hoffbrand and Beck who in 1965, reported 8 out of 24 lupus patients with unexplained dyspnea, decreased lung volumes and ascension of the diaphragmatic domes on radiography [2-4]. The prevalence of SLS in systemic lupus erythematosus varies from 10 to $23 \%$ depending on the series $[5,6]$. SLS has also been reported in other autoimmune diseases including Gougerot-Sjögren syndrome [2-7], rheumatoid arthritis and undifferentiated connectivitis [2-7].

This syndrome usually appears after the diagnosis of lupus, within a variable period ranging from 4 months to 24 years [2-8], in our case SLS appears after one year of disease progression.

The lack of systematic review and research due to the rarity of reported cases continues to be a challenge in understanding of the pathophysiology and development of evidence-based management for this condition. Several mechanisms have been proposed as possible causes including respiratory myopathy, phrenic neuropathy, surfactant deficiency, and pleural adhesions $[1,9]$. Omdal et al., presented a case of bilateral hemidiaphragm elevations and bibasilar atelectasis in a patient who originally had respiratory arrest. The patient was later diagnosed with SLE and shown to have phrenic neuropathy with electromyography and nerve conduction studies. They have also reported diaphragmatic paralysis and myopathy in SLE patients. This patient was treated with cyclophosphamide and methylprednisolone with gradual recovery [10]. Similarly, in our patient, elevation of her left hemidiaphragm could be caused by phrenic neuropathy and diaphragmatic myopathy. Besides elevation of left hemidiaphragm, the restrictive lung pattern in our patient could also be caused by pleurisy.
Treatment in the absence of randomized trials, is not well codified. In most of the published cases, systemic corticosteroid therapy at a dosage of 30 to 60 $\mathrm{mg} /$ day is offered. It is frequently associated with clinical improvement [2-11] which is sometimes rapid [12], functional improvement (increase in total lung capacity, PImax) and also radiological improvement [2]. The use of azathioprine, rituximab, cyclophosphamide and theophylline [7-13] has also been reported in clinical cases with varying results. The need for assisted ventilation is rare [14].

\section{CONCLUSION}

Shrinking lung syndrome is a rare manifestation of SLE. It remains under recognized and poses a diagnostic challenge. Patients with SLE, with normal lung parenchyma, no evidence of pleural effusion, and decrease in total lung capacity on PFT and elevated diaphragm with small lungs on imaging studies should alert the clinicians to the possibility of SLS. Early diagnosis can help in improving the morbidity and mortality. Clinicians need to consider SLS in the differential diagnosis of the patients with SLE who remain symptomatic with declining lung function.

\section{REFERENCES}

1. Choudhury S, Ramos M, Anjum H, Ali M, Surani S. Shrinking lung syndrome: a rare manifestation of systemic lupus erythematosus. Cureus. 2020 May;12(5).

2. Ammara Y, Launoisa C, Perotina JM, Durya S, Servettazb A, Perdua D, Valleranda H, Nardia, J, Boulagnon-Rombic C, Pluotc M, Lebargya F, Desleea G. Lupus erythematosus presenting as severe alveolar hypoventilationand the Shrinking lung syndrome.

3. Duron L, Cohen-Aubart F, Diot E, Borie R, Abad S, Richez C, Banse C, Vittecoq O, Saadoun D, Haroche J, Amoura Z. Shrinking lung syndrome associated with systemic lupus erythematosus: A multicenter collaborative study of 15 new cases and a review of the 155 cases in the literature 
focusing on treatment response and long-term outcomes. Autoimmunity reviews. 2016 Oct 1;15(10):994-1000.

4. Hoffbrand BI, Beck ER. Unexplained dyspnea and shrinking lung in systemic lupus erythematosus. Br Med J. 1965; 1:1273-7.

5. Gibson GJ, Edmonds JP, Hughes GR. Diaphragm function and lung involvement in systemic lupus erythematosus. Am J Med. 1977;63:926-32.

6. Gheita TA, Azkalany GS, El- Fishawy HS, Nour Eldin AM. Shrinking lung syndrome in systemic lupus erythematosus patients; clinical characteristics, disease activity and damage. International journal of rheumatic diseases. 2011 Oct;14(4):361-8.

7. Branger S, Schleinitz $\mathrm{N}$, Gayet $\mathrm{S}$, Veit $\mathrm{V}$, Kaplanski G, Badier M, Magnan A, Harle JR. Shrinking lung syndrome and systemic autoimmune disease. La Revue de medecine interne. 2004 Jan 1;25(1):83-90.

8. Warrington KJ, Moder KG, Brutinel WM. Shrinking lung syndrome in systemic lupus erythematosus. Mayo Clin Proc. 2000; 5:467-72.

9. Deeb M, Tselios K, Gladman DD, Su J, Urowitz MB. Shrinking lung syndrome in systemic lupus erythematosus: a single-centre experience. Lupus. 2018 Mar;27(3):365-71.

10. Omdal R, Roos P, Wildhagen K, Gunnarsson R. Respiratory arrest in systemic lupus erythematosus due to phrenic nerve neuropathy. Lupus. 2004 Oct;13(10):817-9.

11. Orens JB, Martinez FJ, Lynch 3rd JP. Pleuropulmonary manifestations of systemic lupus erythematosus. Rheumatic diseases clinics of North America. 1994 Feb 1;20(1):159-93.

12. Walz-Leblanc BA, Urowitz MB, Gladman DD, Hanly PJ. The" shrinking lungs syndrome" in systemic lupus erythematosus--improvement with corticosteroid therapy. The Journal of rheumatology. 1992 Dec 1;19(12):1970-2.

13. Meinicke H, Heinzmann A, Geiger J, Berner R, Hufnagel M. Symptoms of shrinking lung syndrome reveal systemic lupus erythematosus in a 12- year- old girl. Pediatric pulmonology. 2013 Dec;48(12):1246-9.

14. Piccolo-Daher S, Magalhães E. Anesthesia in patient with shrinking lung syndrome: case report. Revista brasileira de anestesiologia. 2012 Apr;62(2):277-80. 